\title{
Semi-conductivity Transition in Silicon Nanowires by Hole Transport Layer
}

Awad Shalabny, ${ }^{1}$ Francesco Buonocore, ${ }^{2}$ Massimo Celino, ${ }^{2}$ Gil Shalev, ${ }^{3}$ Lu Zhang, ${ }^{4}$ Weiwei Wu, ${ }^{4}$ Peixian Li, ${ }^{4}$ Jordi Arbiol, ${ }^{5}$ and Muhammad Y. Bashouti ${ }^{1,6^{*}}$

${ }^{1}$ Department of Solar Energy and Environmental Physics, Swiss Institute for Dryland Environmental and Energy Research, J. Blaustein Institutes for Desert Research, Ben-Gurion University of the Negev, Midreshset Ben-Gurion, Building 26, 8499000, Israel.

${ }^{2}$ ENEA, C. R. Casaccia, via Anguillarese 301, 00123 Rome, Italy.

${ }^{3}$ School of Electrical \& Computer Engineering, Ben-Gurion University of the Negev, POB653, Beer-Sheva 8410501, Israel.

${ }^{4}$ School of Advanced Materials and Nanotechnology, Interdisciplinary Research Center of Smart Sensors, Xidian University, Shaanxi, 710126, PR. China

5 Institució Catalana de Recerca i Estudis Avançats (ICREA) and Institut de Ciència de Materials de Barcelona, ICMAB-CSIC, 08193, Bellaterra, CAT, Spain.

${ }^{6}$ The IISe-Katz Institute for Nanoscale Science \& Technology, Ben-Gurion University of the Negev, POB 653, BeerSheba Campus, Building 51, 8410501, Israel.

*e-mail:Bashouti@bgu.ac.il

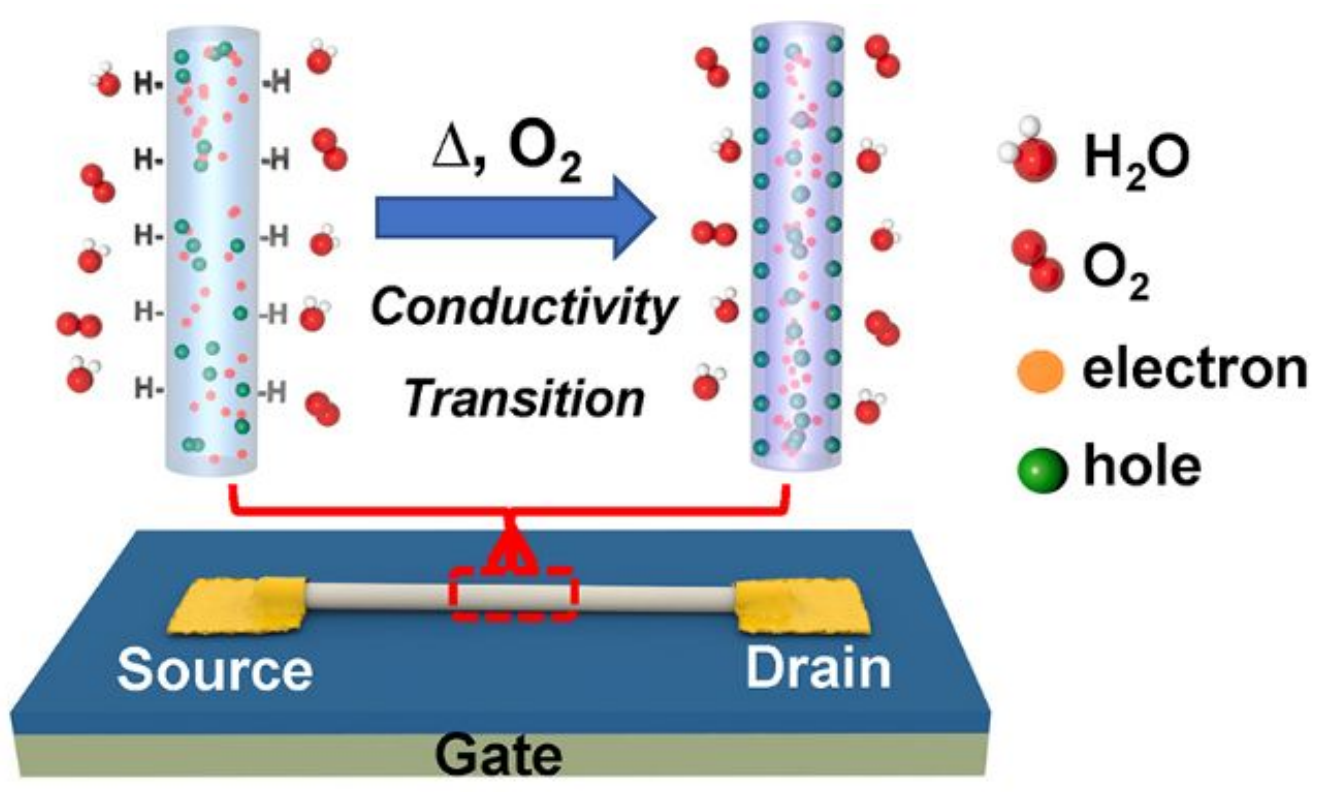




\begin{abstract}
Nanowires surface is a source of interest mainly for electrical prospects. Thus, different surface chemical treatments were carried to develop recipes to control the surface effect. In this work, we succeed to shift and tune the semi-conductivity of Si nanowire based device form n- to p-type. This accomplished by generating hole transport layer at the surface by using an electrochemical reaction-based non-equilibrium position to enhance the impact of the surface charge transfer. This completed by applying different annealing pulses at low temperature (below $400{ }^{\circ} \mathrm{C}$ ) to reserve the hydrogen bonds at the surface. After each annealing pules, the surface characterized by XPS, Kelvin Probe measurements and conductivity measured by FET based single Si NW. the mechanism and conclusion were supported experimentally and theoretically. To this end, this strategy has been demonstrated as an essential tool which could pave a new road for regulating the semi-conductivity and for other low-dimensional nano-materials.
\end{abstract}

\title{
Keywords
}

Silicon nanowire, Surface doping, Enhanced surface reaction, Hole transport layer, Semiconductivity transition. 
Semi-conductors with $\mathrm{n}$ and/or $\mathrm{p}$ types are the basis of semi-conductive devices that build the whole electronic industry. ${ }^{1}$ The conventional strategy to control the semi-conductive types, i.e., $n$ type (electron mobility) and p type (hole mobility), is doping the intrinsic semi-conductor with heterogeneous elements which contain different valence electrons. For example, Si doped with $\mathrm{P}$ and $\mathrm{B}, \mathrm{GaN}$ doped with $\mathrm{Si}$ and $\mathrm{Mg}$, etc., in nature, which is the regulation of defects and insertion of doping energy levels in the intrinsic band gap of body semi-conductors. ${ }^{2,3}$ When the semiconductors come into nanoscale, this process is also influenced and/or amplified by surfaces as another kind of ultra-important defects in nano-materials, has dramatically different properties comparing with bulk materials, however, rare to be reported directly regulating the semiconductive types. ${ }^{4,5}$

An effective strategy to realizes this process is based on surface charge transfer doping (SCTD) which terminate the surface with atoms/molecules producing a transport layer at the surface. ${ }^{6-8}$ To this end, we are able to tune not only the doping profile but also the surface potential and band bending. ${ }^{1,8-11}$ To date, these surface modifications were performed under equilibrium reaction conditions which could limit the electron withdrawing or donating by the grafted atoms/molecules. ${ }^{12-14}$ However, here we applied annealing pulses to disturb the equilibrium reaction, simulating the Haber-Bosch process but at the nano scale. According to le Chatelier principle, when an equilibrium is perturbed, the equilibrium position moves to counteract the perturbation and, in this way, we expect that electrons withdrawing/donating can be enhanced.

We illustrate this idea on the silicon nanowires (SiNWs), because the one-dimensional channel is suitable for carriers' mobility while the optical absorption cross-section provides a strong resonant field enhancement of the impinging illumination ${ }^{15,16}$. Various thermal oxidation surfaces with different coverages and thickness, are greatly important in traditional surface passivation 
process commonly employed in the semiconductor industry for minimizing the surface affects, Inc., surface states and dangling bonds (reported densities about $10^{10} \mathrm{~cm}^{-3}$ ). Usually, the annealing temperature in these procedures was chosen to be high (more than $600{ }^{\circ} \mathrm{C}$ ) in order to achieve a direct interaction between the Si dangling bond with the ionized oxygen from the ambient and to decrease fixed charge density. ${ }^{17}$ However, in our case, we chose an annealing temperature less than $400{ }^{\circ} \mathrm{C}\left(440{ }^{\circ} \mathrm{C}\right.$ is needed to break $\mathrm{Si}-\mathrm{H}$ bond). Therefore, we force the oxidation mechanism of the $\mathrm{Si}$ NWs to rely on $\mathrm{Si}-\mathrm{H}$ bond and $\mathrm{O}_{2}$ molecules to go through a redox couple reaction controlled by SCTD, avoiding producing a fixed charge (e.g. producing $\mathrm{Si}-\mathrm{O}^{+}$complexes) which is an additional advantage of this technique. As a result, the semi-conductivity of silicon NWs were able to be tuned between $\mathrm{n}$ and $\mathrm{p}$ semi-conductive types due to the charge transfer process.

In this paper we show the transition between $\mathrm{n}$ and $\mathrm{p}$ semi-conductive types have been successfully achieved by disturbing the reaction equilibrium on the Si NWs surface through a simple pulse annealing approach. Their mechanisms are well studied theoretically and experimentally. This strategy has been demonstrated as an essential tool when the Si based electronic device goes to nanoscale, which could pave a new road for regulating the semiconductive types and for other low-dimensional nano-materials.

The Si NWs are single crystalline, exhibiting [112] and were grown by the VLS method with an average length and diameters of $3.51 \pm 0.26 \mu \mathrm{m}$ and $65 \pm 25 \mathrm{~nm}$, respectively (See Supplementary Information, SI). To start with identical surfaces after each (non)equilibrium step, a full Si-H monolayer (ML) on the VLS grown Si NWs surfaces was produced by immersing the Si NWs samples in buffered HF solution $(\mathrm{pH}=5)$ for 30 sec (See SI). Afterwards, we applied a series of annealing pulses ( 4 cycles with temperatures ranging from $100{ }^{\circ} \mathrm{C}$ to $400{ }^{\circ} \mathrm{C}$, See SI and Figure 1a). These conditions are and limited by the stability of the $\mathrm{Si}-\mathrm{H}$ bonds (up to $440{ }^{\circ} \mathrm{C}$ ). As 
reference data, we also obtained the same $\operatorname{ML}(1$ monolayer of oxide is define when the emission ratio between the $\mathrm{SiOx}$ and $\mathrm{Si} 2 \mathrm{p}$ in XPS equal to 0.21$)^{18}$ intensity under equilibrium reaction conditions. Compared to the composition of the oxide layer (at $\mathrm{ML}<1$ ), the $\mathrm{SiO}_{2}$ content increased to $66.97 \%$ while the sub-oxide content decreased to $27.3 \%$ at ML>1.
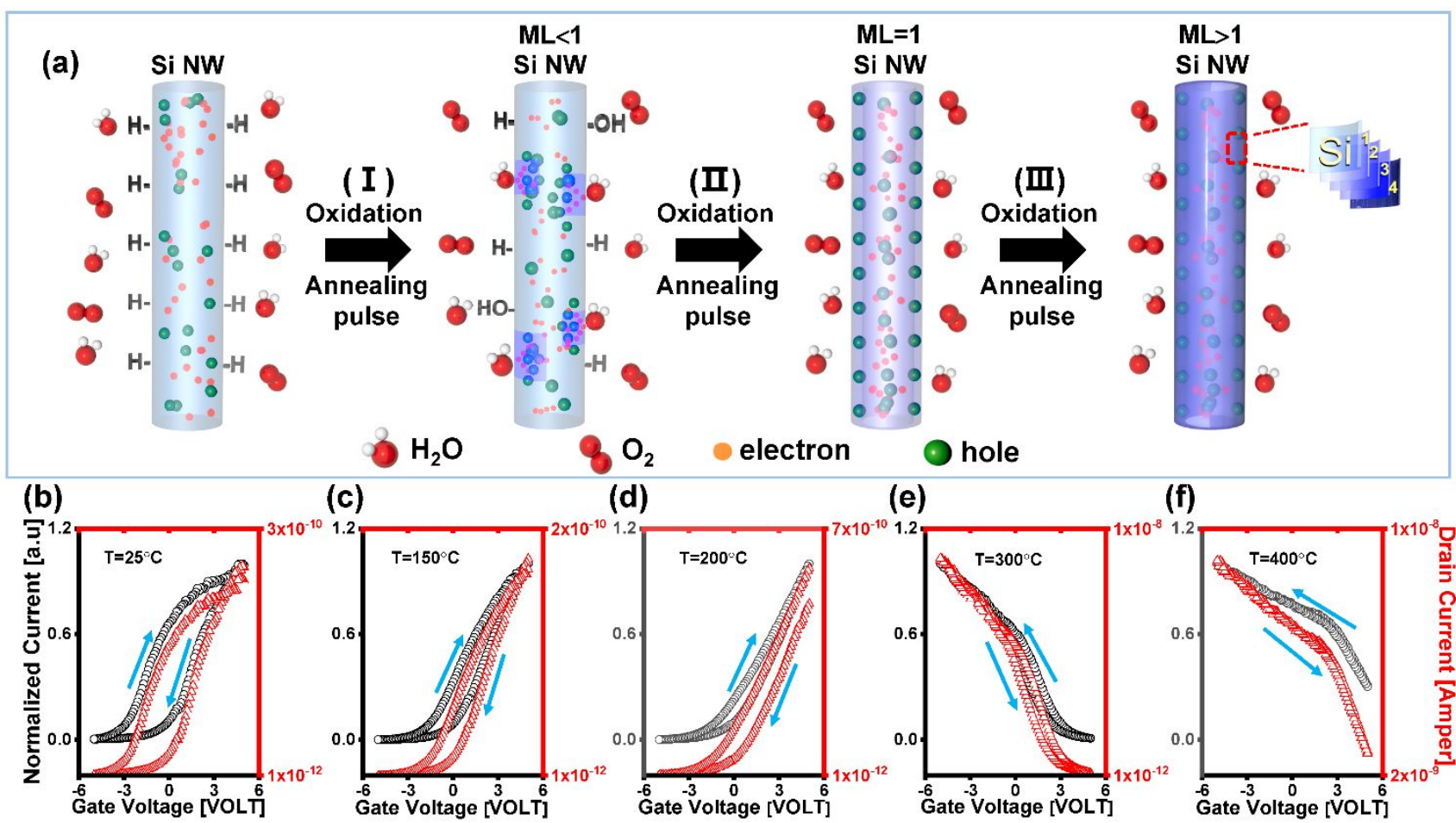

(c)

(d)
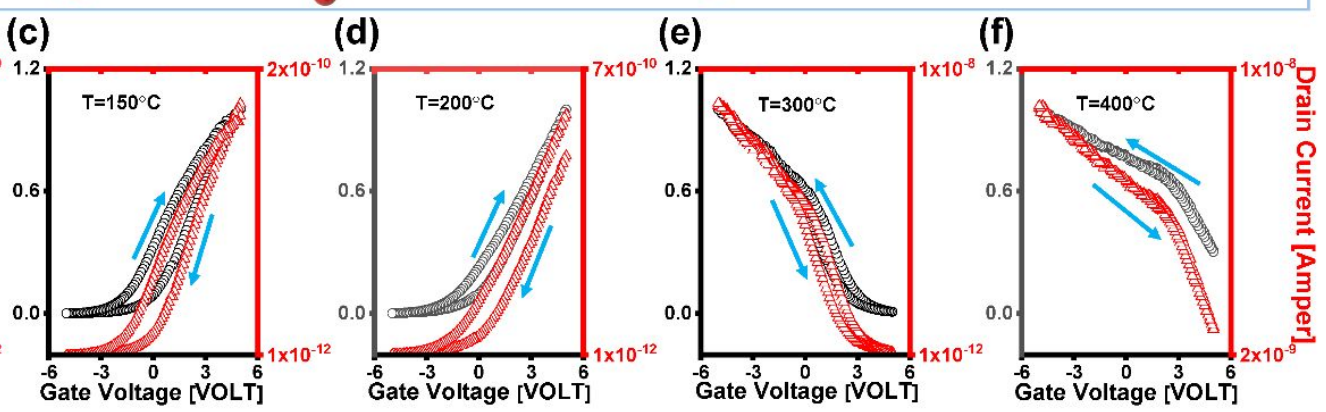

Figure 1. (a) is a schematic diagram for the annealed pulses process along the different stages from $M L=0$, to $M L$ $<1$ and $M L>1$. $\left(\mathrm{b}-\mathrm{f}\right.$ ) Left hand Normalized current values (current value/ $\mathrm{I}_{\text {on }}$ ) for $0.6,1.35,2,2.7$ and 4.6 oxide $\mathrm{ML}$, respectively. The Hysteresis were measured on single Si NWs based FET, the right hand is the actual current, the blue arrows represent the direction of the voltage change.

To reveal the electrical properties of the Si NWs after the annealing pulses, a current-voltage diagram was used (see Figure 1b-f), measurement conducted in vacuum condition. A single $\mathrm{Si}$ NW was used as the conductive channel of a Field Effect Transistor (FET) between two gold electrodes representing the source and drain. The interface composes of $\mathrm{Si} / \mathrm{SiO} / \mathrm{Ti} / \mathrm{Au}$ producing Schottky barrier, therefore, the charge extraction process takes place in the electrodes (see SI) ${ }^{19}$. Two main results were clearly observed: (i) hysteresis loop, which decreases with increasing the 
perturbation pulses (Figure 1b-f), and (ii) a transition from n-type conductivity to p-type conductivity after forming a full monolayer. Therefore, a hole accumulation layer is produced at the surface and a dynamical surface states appeared during the surface modification.

The transition in the conductivity from n-type to p-type is attributed mainly to two processes: first, SCTD via the redox reaction, second, the perturbation of the reaction equilibrium. The relation between these two processes is shown by the redox couple based chemical reaction $\left(\mathrm{O}_{2}+4 \mathrm{H}^{+}+\right.$ $\left.4 \mathrm{e}^{-} \leftrightharpoons 2 \mathrm{H}_{2} \mathrm{O}, \mathrm{V}=-5.1 \mathrm{eV}\right)$. To this end, the $\mathrm{Si}-\mathrm{H}$ consumes one electron to produce one water molecule ${ }^{12}$ and goes through a transition state that can trap electrons. Evaporating the produced water molecules from the surface leaves vacancies at the Si surface with a lack of an electron, and as a result, a hole transport layer is produced at the surface. Thus, P-type doping is occurring and p conductivity can be detected and a change in the current value by 3 orders of magnitude was found as shown in Figure 1, for carrier concentration see table S1 in SI. It is worth to mention that by etching the surface again, the conductivity and the fermi level back to the same initial position, in addition, stability measurement at ambient condition has been conducted, see supporting information. Alternatively, when maintaining the equilibrium conditions, electrons can transfer from the Si surface to the water molecule and vice versa, as a result, no net charge transfer is occurring and a constant fermi level can be detected (Figure 3c). Thus, the reaction kinetics is attributed to the production of surface transport layer that controls the overall behavior of the device. With this mind, the raising of temporary dynamical surface states that can be charged and discharged under an applied bias can explain the observed hysteresis. ${ }^{20,21}$ To explain the hysteresis, a First principles calculation has been employed (see SI) based on density functional theory (DFT) using the climbing-image nudged elastic band (NEB). This method was used to assess the difference in the charging and discharging processes of the trap center to explain the physical 
mechanism behind the observed hysteresis loops. The formation energy for neutral, positive and negative transition states was calculated as a function of the Fermi level. In minimal energy configuration, the hydrogen trap in the neutral state is localized at the center of an interstitial void. In the positively charged state, hydrogen is coordinated with an oxygen atom, while in the negatively charged state, the hydrogen atom is bound to a $\mathrm{Si}$ atom. A nonsymmetrical barrier was found, with the transition state along the energy pathway in between $\mathrm{Si}-\mathrm{H}$ states (Figure 2a), with a reaction coordinate equal to 0.5 , and $\mathrm{Si}-\mathrm{OH}^{+}$, with a reaction coordinate equal to 1 , which can be overcome via an applied bias. The formation energy is written as $\mathrm{E}_{\mathrm{f}}=\mathrm{E}_{\mathrm{TOT}}-\left(\mathrm{E}_{\mathrm{UNP}}+\mathrm{E}_{\mathrm{H} 2} / 2\right)-\mathrm{n} \mu$, where $\mathrm{E}_{\mathrm{TOT}}$ and $\mathrm{E}_{\mathrm{UNP}}$ are the total energies of the system with a hydrogen trap and the unperturbed system without a trap, respectively, and $\mathrm{E}_{\mathrm{H} 2}$ is the total energy of the isolated hydrogen molecule, with $n=0$ for a neutral system and $n=+1$ and -1 for negatively and positively charged systems respectively (Figure $2 \mathbf{b}$ ). The electron Fermi level $\mu$ is related to the valence-band edge $E_{V}$ in the unperturbed system according to the equation $\mu=\mathrm{EV}+\Delta \mathrm{V}+\mathrm{x}$, where $\mathrm{x}$ varies from the valence $(\mathrm{x}=0 \mathrm{eV})$ to the conduction $(\mathrm{x}=5.6$ $\mathrm{eV}$ ) band edge and $\Delta \mathrm{V}$ accounts for the shift of the average potential of the system with the trap relative to the unperturbed system. It was calculated that the production of two states of opposite charge in the middle of the gap (when they have the same formation energy) corresponds to a gain of energy of $2 \mathrm{E}+/=2.7 \mathrm{eV}$. The formation energy of the positive state is lower than the formation energy of the negative state when the Fermi level is above $\chi^{+/-}=2.8$ $\mathrm{eV}$. In addition, at a Fermi level above $4.2 \mathrm{eV}$, the formation energy of the negative state becomes greater than 0, i.e. formation of the negative state is not favorable based on thermodynamic rules. This reasoning indicates that the only states that can appear in our system above $4.2 \mathrm{eV}$ (binding energy of $99.6 \mathrm{eV}$, corresponding to $0.88 \mathrm{eV}$ in as explained later Figure 3b) are the neutral $(\mathrm{Si}-\mathrm{H})$ and positive $\left(\mathrm{Si}-\mathrm{OH}^{+}\right)$states (Figure $\left.2 \mathrm{a} \& \mathrm{~b}\right)$. This result emphasizes that 
the transition from a positive to a neutral state and vice versa is responsible for the hysteresis loop as expected (the Fermi level in our system changes from 4.2 to $4.4 \mathrm{eV}$, Figure $2 \mathrm{~b}$ ). Specifically, the neutral-state to positive-state barrier is approximately $0.82 \mathrm{eV}$, whereas the opposite barrier (from positive to neutral) is $0.89 \mathrm{eV}$ (Figure 2a). These energy barriers are much lower than those calculated for $\mathrm{H}$ bridge defects and comparable to those calculated for hydroxyl-E' defects for the transition from neutral to positively charged states in amorphous silicon oxide. ${ }^{22}$ Calculated differences in energy barriers $(0.07 \mathrm{eV})$, represent the difference in the charging and discharging processes, and lead to hysteresis loops in the I-V graph, as shown in Figure 1b-e.
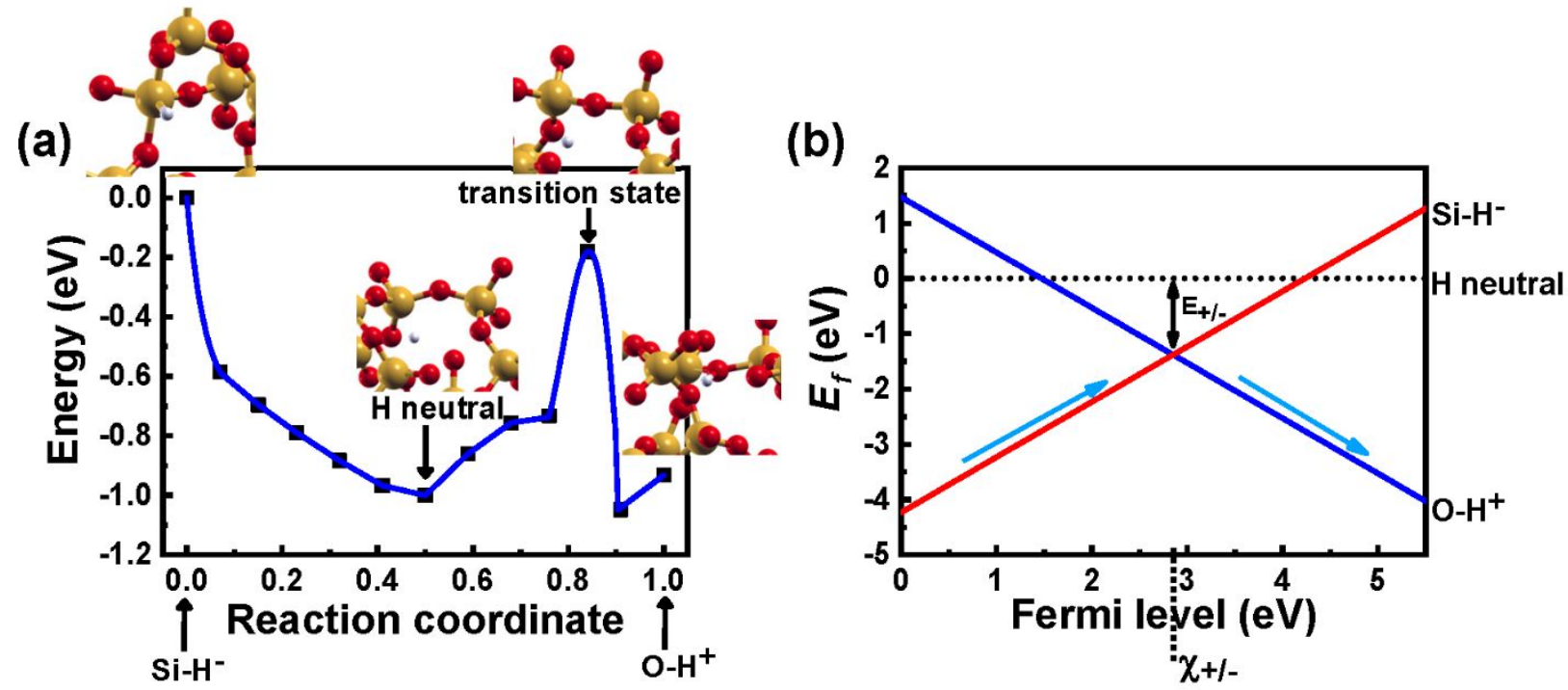

Figure 2. Charge trap states at the surface. (a) Calculated Energy pathways between negative and neutral and between neutral and positive states of charge of the hydrogen trap in the silicon oxide, where the energy of the states (black squares) is interpolated as the blue curve for visualization purposes. Yellow spheres are $\mathrm{Si}$, white spheres are $\mathrm{H}$, and red spheres are $\mathrm{O}$ atoms. (b) Formation energy for different charge states as a function of the Fermi level (the red line is the formation energy of $\mathrm{Si}-\mathrm{H}^{-}$, the blue line is the formation energy of $\mathrm{Si}-\mathrm{OH}^{+}$, the black dotted line is the formation energy of the neutral $\mathrm{H}$ trap, and the blue arrows track the minimal formation energy path).

To investigate the fermi level of the surface we measured the XPS after each annealing pulse. Interestingly, the $\mathrm{Si} 2 \mathrm{p}_{3 / 2}$ emissions shifted to a lower binding energy, indicating for increase in the positive charge of the Si atoms, as illustrated in Figure 3a\&b. It's worth to mention, continuing the oxidation process (above 4.6 ML) will lead to surface charging (See SI) and an increase in the 
binding energy will occur, thus, we stop the oxidation process at 4.6 ML, this value presents the upper limit of such surface doping. This unforeseen shift suggests that the surface chemistry proceeds via the suggested mechanism and affects the electronic properties.
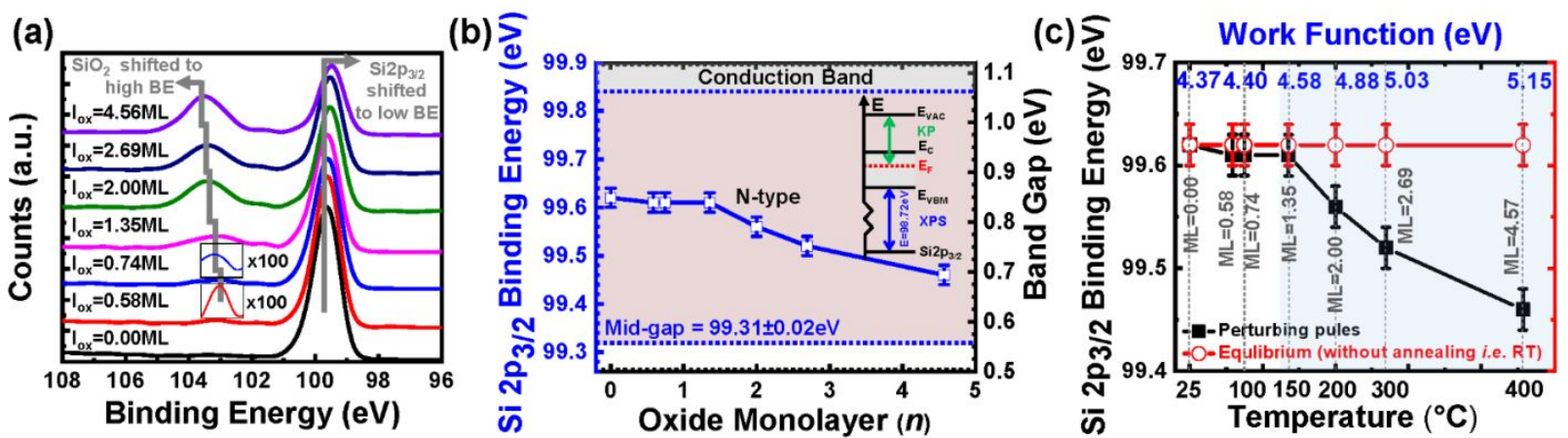

Figure 3. (a) Si2 $\mathrm{p}_{3 / 2}$ emission of the Si NW surface measured by XPS, which shifted to a lower binding energy by the annealing pulses. The $\mathrm{x} 100$ represents 100 -time magnification of the oxide emission indicating the formation of suboxides. (b) Si2 $\mathrm{p}_{3 / 2}$ binding energies measured relative to the Fermi level and converted to the SFL position with respect to the valence band edge $E_{V}$ of Si. The Si2p emission, (left) is the corresponding SFL according to our model, the insight is the band diagram of the emission. (c) Si2 $\mathrm{p}_{3 / 2}$ binding energy with and without annealing pulses. The red line represents the $\mathrm{Si} 2 \mathrm{p}_{3 / 2}$ binding energy position without annealing (red line). The black line represents the $\mathrm{Si} 2 \mathrm{p}_{3 / 2}$ binding energy position after annealing pulses. The transparent light blue represents $\mathrm{ML}>1$.

To measure this shift precisely, we aligned the $\mathrm{Sip}_{3 / 2}$ emission with the Surface Fermi Level (SFL). At first step, we extract the Si2 $\mathrm{p}_{3 / 2}$ emission from the Si2p emission by splitting into $\mathrm{Si} 2 \mathrm{p}_{1 / 2}$ and $\mathrm{Si} 2 \mathrm{p}_{3 / 2}$ emissions (Figure 3a). Then based on the fact that $\mathrm{Si} 2 \mathrm{p}$ has a constant binding energy relative to the valence band, the measured difference between the obtained SFL and energy of the valence-band maximum $\left(\mathrm{E}_{\mathrm{V}}-\mathrm{E}_{\mathrm{F}}\right.$; when $\mathrm{p}^{++}$used) and conduction band minimum $\left(\mathrm{E}_{\mathrm{C}}\right.$; when $\mathrm{n}^{++}$ used) were $98.72 \pm 0.02 \mathrm{eV}$ and $99.84 \pm 0.02 \mathrm{eV}$ respectively. These values agree for bulk $\mathrm{Si}$ as previously published by our group, Ristein and Himpsel..$^{7,9,23,24}$ The difference between the SFL in the two samples, $\left(\mathrm{p}^{++} \& \mathrm{n}^{++}\right)$, equals $1.1 \pm 0.04 \mathrm{eV}$, which is the difference between $\mathrm{E}_{\mathrm{C}}$ and $\mathrm{E}_{\mathrm{V}}$ and represents the energy gap and midgap is at $99.31 \pm 0.03 \mathrm{eV}$ (Figure 3b). Anticipating the corresponding edge values of the $\mathrm{E}_{\mathrm{C}}, \mathrm{E}_{\mathrm{V}}$ and midgap with the relevant $\mathrm{Sip}_{3 / 2}$ positions, it is possible to align the binding energy with the SFL scales and the shift direction. We can infer that the Si2 $\mathrm{p}_{3 / 2}$ of the freshly etched sample (i.e. Si-H surface) is n-type with a binding energy of $99.6 \pm 0.02 \mathrm{eV}$, 
which corresponds to the SFL of $0.88 \mathrm{eV}$ above the valence band. In the equilibrium regime, the SFL maintains its value without any shift (Figure 3c). However, as the annealing pulses start (from $100^{\circ} \mathrm{C}$ to $400^{\circ} \mathrm{C}$ ), the equilibrium is perturbed on the VLS-grown Si NWs, and a gradual shift of the SFL is observed from $99.62 \pm 0.02 \mathrm{eV}$ to $99.42 \pm 0.02 \mathrm{eV}$ (Fig. 3b). Note that since the $\mathrm{Si}_{2} \mathrm{p}_{3 / 2}$ signal is an average of the $\mathrm{Si}-\mathrm{Si}$ bonds at the surface and $\mathrm{Si}-\mathrm{Si}$ bonds of the bulk, due to the sampling depth for $3 \mathrm{~nm}$, the total shift, $200 \mathrm{meV}$, can be higher showing an inversion from n-type to p-type. As the shift is to lower binding energy, this indicates for a positive charge accumulation at the Si surface i.e. more p-type character as was shown in the conductivity measurements, in addition, etching the oxide layer that produced in non-equilibrium condition take us back to the initial Si2p emission and n-type conductivity.

To further assist this conclusion, we measured the work function $(\Phi)$ in-situ using a $\mu$-KP in the $\mathrm{Si} 2 \mathrm{p}_{3 / 2}$ emission area, to have a schematic study for the same spot that was measured by XPS. The surface electron affinity $(\chi)$ was calculated by $\chi=\Phi-E_{g}+\left(E_{F}-E_{V}\right)$, where $E_{g}$ is the band gap (1.12 $\mathrm{eV}), \mathrm{E}_{\mathrm{F}}$ is the SFL and $\mathrm{E}_{\mathrm{V}}$ is the valence band maximum. ${ }^{11}$ As illustrated in Figure 3c, the higher the perturbation (annealing pulses), the more oxide layer grows, the higher $\Phi$ and $\chi$ are, and the lower the SFL is. Specifically, prior to oxide thickness of $\mathrm{ML}<1$, slight increases were observed in $\Phi(\Delta \Phi \sim 0.1 \pm 0.02)$ and $\chi(\Delta \chi \sim 0.11 \pm 0.02 \mathrm{eV})$ and a marginal change in the SFL position $(<$ $20 \mathrm{meV}$ ). However, after perturbing the reaction equilibrium beyond $\mathrm{ML}>1, \Phi$ and $\chi$ increases by $0.65 \pm 0.03 \mathrm{eV}$ and $0.47 \pm 0.03 \mathrm{eV}$, respectively, and the SFL notably decreases by $200 \pm 0.02$ meV (Figure 3c). The nonsymmetrical increase in the $\chi$ and $\Phi$, indicates again our conclusion from the hysteresis and the theoretical calculation that we have a charge transfer and as $\Delta \Phi>\Delta \chi$ we have an electron depletion i.e. SCTD process. 
In summary, we demonstrate that pulse annealing approach for disturbing the reaction equilibrium on the SiNW surface has been successfully applied. The switch of $n$ and $p$ semiconductive types is achieved, and its mechanism are well studied theoretically and experimentally. The investigation by first principles calculations revealed that $\mathrm{Si}-\mathrm{O}-\mathrm{Si}, \mathrm{Si}-\mathrm{H}$ and $(\mathrm{Si}-\mathrm{OH})^{+}$surface functionalization is present on the surface at the initial stage of oxidation and that the traps charge/discharge process can explain the hysteresis loops observed in the I-V plots. The reported results quantify the significant role of surface chemistry kinetics in nano-regime on the electronic properties of nanomaterials and illustrate important insights into the impact of surface properties for integrating nano-semiconductor based devices into nanotechnology research and industry.

\section{Supporting Information}

The Supporting Information is available free of charge via the internet at http://pubs.acs.org.

Synthesis of Si NWs, Si NWs hydrogen termination, Oxide states growth on H-Si NWs, Device Fabrication, Density functional theory calculations, Characterization of Si NWs, Work function measurements by the Kelvin probe method, Electron Hole concentration calculation, Stability of the Si NW in ambient condition and Surface charging.

\section{Notes}

The authors declare no competing financial interest.

\section{Author Information}

\section{Corresponding Author}

Muhammad Y. Bashouti - Department of Solar Energy and Environmental Physics, Swiss Institute for Dryland Environmental and Energy Research, J. Blaustein Institutes for Desert Research, Ben-Gurion University of the Negev, Midreshset Ben-Gurion, building 26, 8499000, Israel; The IISe-Katz Institute for Nanoscale Science \& Technology, Ben-Gurion University of the Negev,POB 653,Beer-Sheba Campus, Building 51,8410501,Israel.E-mail: bashouti@bgu.ac.il; ORCID: https://orcid.org/0000-0002-0371-7088

\section{Authors}

Awad Shalabny - Department of Solar Energy and Environmental Physics, Swiss Institute for Dryland Environmental and Energy Research, J. Blaustein Institutes for Desert Research, Ben- 
Gurion University of the Negev, Midreshset Ben-Gurion, Building 26, 8499000, Israel. E-mail: awads@bgu.ac.il; ORCID: https://orcid.org/0000-0002-7012-6210

Francesco Buonocore - ENEA, C. R. Casaccia, via Anguillarese 301, 00123 Rome, Italy. Email: francesco.buonocore@enea.it; ORCID: https://orcid.org/0000-0001-5028-4468

Massimo Celino - ENEA, C. R. Casaccia, via Anguillarese 301, 00123 Rome, Italy. Email: massimo.celino@enea.it; ORCID: http://orcid.org/0000-0002-9707-991X

Gil Shalev - School of Electrical \& Computer Engineering, Ben-Gurion University of the Negev, POB653, Beer-Sheva 8410501, Israel.Email:glshalev@bgu.ac.il

Lu Zhang - School of Advanced Materials and Nanotechnology, Interdisciplinary Research Center of Smart Sensors, Xidian University, Shaanxi, 710126, PR. China. Email: luzha@post.bgu.ac.il

Weiwei Wu - School of Advanced Materials and Nanotechnology, Interdisciplinary Research Center of Smart Sensors, Xidian University, Shaanxi, 710126, PR. China. Email: wwwu@xidian.edu.cn

Li Peixian - School of Advanced Materials and Nanotechnology, Interdisciplinary Research Center of Smart Sensors, Xidian University, Shaanxi, 710126, PR. China. Email: pxli@mail.xidian.edu.cn

Jordi Arbiol - Institució Catalana de Recerca i Estudis Avançats (ICREA) and Institut de Ciència de Materials de Barcelona, ICMAB-CSIC, 08193, Bellaterra, CAT, Spain. Email: arbiol@icrea.cat

\section{Author Contributions}

The manuscript was written through contributions of all authors. All authors have given approval to the final version of the manuscript.

\section{Acknowledgements}

MB thanks the MAOF Grant from the Council for Higher Education in Israel for new faculty members. AS is appreciative of the institutional scholarships for Ph.D. students they received from Ben-Gurion University of the Negev. W.W. thanks the fundamental research funds the central universities (JC2002). Lu Zhang thanks the support of postdoctoral fellowship from the Jacob Blaustein Center for Scientific Cooperation, the National Natural Science Foundation of China (Grant No. 61904134), the National Natural Science Foundation of Shaanxi Province (Grant No. 2019JQ-291). The computing resources and the related technical support used for this work have been provided by CRESCO/ENEAGRID High Performance Computing infrastructure and its staff, along the others S. Migliori and S. Giusepponi. ENEA acknowledge funding from the European Union's Horizon 2020 research and innovation programme under grant agreement No 
824158 (EoCoE-II). JA acknowledge funding from Generalitat de Catalunya 2017 SGR 327 and the Spanish MINECO coordinated project ENE2017-85087-C3. ICN2 is supported by the Severo Ochoa program from Spanish MINECO (Grant No. SEV-2017-0706) and is funded by the CERCA Programme / Generalitat de Catalunya.

\section{REFERENCES}

(1) Hoffmann, S.; Bauer, J.; Ronning, C.; Stelzner, T.; Michler, J.; Ballif, C.; Sivakov, V.; Christiansen, S. H. Axial P-n Junctions Realized in Silicon Nanowires by Ion Implantation. Nano Lett. 2009, 9, $1341-1344$.

(2) Mirabella, S.; De Salvador, D.; Napolitani, E.; Bruno, E.; Priolo, F. Mechanisms of Boron Diffusion in Silicon and Germanium. J. Appl. Phys. 2013, 113 (3).

(3) Brotzmann, S.; Bracht, H. Intrinsic and Extrinsic Diffusion of Phosphorus, Arsenic, and Antimony in Germanium. J. Appl. Phys. 2008, 103 (3).

(4) Cahen, D.; Kahn, A. Electron Energetics at Surfaces and Interfaces: Concepts and Experiments. Adv. Mater. 2003, 15 (4), 271-277.

(5) Cui, Y.; Lieber, C. M. Functional Nanoscale Electronic Devices Assembled Using Silicon Nanowire Building Blocks. Science (80-. ). 2001, 291 (5505), 851-853.

(6) Zhang, X.; Shao, Z.; Zhang, X.; He, Y.; Jie, J. Surface Charge Transfer Doping of Low-Dimensional Nanostructures toward High-Performance Nanodevices. Adv. Mater. 2016, 28 (47), 10409-10442.

(7) Bashouti, M. Y.; Paska, Y.; Puniredd, S. R.; Stelzner, T.; Christiansen, S.; Haick, H. Silicon Nanowires Terminated with Methyl Functionalities Exhibit Stronger $\mathrm{Si}-\mathrm{C}$ Bonds than Equivalent 2D Surfaces. Phys. Chem. Chem. Phys. 2009, 11, 3845-3848.

(8) He, Y.; Xia, F.; Shao, Z.; Zhao, J.; Jie, J. Surface Charge Transfer Doping of Monolayer Phosphorene via Molecular Adsorption. J. Phys. Chem. Lett. 2015, 6 (23), 4701-4710.

(9) Bashouti, M. Y.; Tung, R. T.; Haick, H. Tuning the Electrical Properties of Si Nanowire Field-Effect Transistors by Molecular Engineering. Small 2009, 5 (23), 2761-2769.

(10) Chen, W.; Qi, D.; Gao, X.; Wee, A. T. S. Surface Transfer Doping of Semiconductors. Progress in 
Surface Science. 2009, pp 279-321.

(11) Leung, T. C.; Kao, C. L.; Su, W. S.; Feng, Y. J.; Chan, C. T. Relationship between Surface Dipole, Work Function and Charge Transfer: Some Exceptions to an Established Rule. Phys. Rev. B 2003, $68(19)$

(12) Yuan, G. D.; Zhou, Y. B.; Guo, C. S.; Zhang, W. J.; Tang, Y. B.; Li, Y. Q.; Chen, Z. H.; He, Z. B.; Zhang, X. J.; Wang, P. F.; Bello, I.; Zhang, R. Q.; Lee, C. S.; Lee, S. T. Tunable Electrical Properties of Silicon Nanowires via Surface-Ambient Chemistry. ACS Nano 2010, 4 (6), 3045-3052.

(13) Van Laer, R.; Kuyken, B.; Van Thourhout, D.; Baets, R. Interaction between Light and Highly Confined Hypersound in a Silicon Photonic Nanowire. Nat. Photonics 2015, 9 (3), 199-203.

(14) Field, N.; Paska, Y.; Stelzner, T.; Christiansen, S.; Haick, H. Enhanced Sensing of Nonpolar Volatile Organic Compounds by Silicon. ACS Nano 2011, 5 (7), 5620-5626.

(15) Tian, B.; Zheng, X.; Kempa, T. J.; Fang, Y.; Yu, N.; Yu, G.; Huang, J.; Lieber, C. M. Coaxial Silicon Nanowires as Solar Cells and Nanoelectronic Power Sources. Nature 2007, 449 (7164), 885-889.

(16) Bandaru, P. R.; Pichanusakorn, P. An Outline of the Synthesis and Properties of Silicon Nanowires. Semicond. Sci. Technol. Technol. 2010, 25 (100), 024003.

(17) Raider, S. I. On the Nature of Fixed Oxide Charge. J. Electrochem. Soc. 1978, 125 (4), 629.

(18) Webb, L. J.; Nemanick, E. J.; Biteen, J. S.; Knapp, D. W.; Michalak, D. J.; Traub, M. C.; Chan, A. S. Y.; Brunschwig, B. S.; Lewis, N. S. High-Resolution X-Ray Photoelectron Spectroscopic Studies of Alkylated Silicon(111) Surfaces. J. Phys. Chem. B 2005, 109 (9), 3930-3937.

(19) Koren, E.; Elias, G.; Boag, A.; Hemesath, E. R.; Lauhon, L. J.; Rosenwaks, Y. Direct Measurement of Individual Deep Traps in Single Silicon Nanowires. Nano Lett. 2011, 11 (6).

(20) Kim, W.; Javey, A.; Vermesh, O.; Wang, Q.; Li, Y.; Dai, H.; V, S. U. Hysteresis Caused by Water Molecules in Carbon Nanotube Field-Effect Transistors. Nano Lett. 2003, 3 (D), 193-198.

(21) Paska, Y.; Haick, H. Interactive Effect of Hysteresis and Surface Chemistry on Gated Silicon Nanowire Gas Sensors. ACS Appl. Mater. Interfaces 2012, 4 (5), 2604-2617.

(22) Wimmer, Y.; El-Sayed, A.-M.; Gös, W.; Grasser, T.; Shluger, A. L. Role of Hydrogen in Volatile 
Behaviour of Defects in $\mathrm{SiO}_{2}$-Based Electronic Devices. Proc. R. Soc. A Math. Phys. Eng. Sci. 2016, 472 (2190), 20160009.

(23) Himpsel, F. J.; Hollinger, G.; Pollak, R. A. Determination of the Fermi-Level Pinning Position at Si(111) Surfaces. Phys. Rev. B 1983, 28 (12), 7014-7018.

(24) Bashouti, M. Y.; Sardashti, K.; Christiansen, S. H. Early Stages of Oxide Growth in H-Terminated Silicon Nanowires : Determination of Kinetic Behavior and Activation Energy. Phys. Chem. Chem. Phys. 2012, 14, 11877-11881. 